\title{
AUTOMATION AND ROBOTIC ASSISTED LIVING
}

\author{
Thomas Bock \\ Technische Universität München, Munich \\ thomas.bock@bri.ar.tum.de \\ Thomas Linner, Gerhard Fabritz \\ Technische Universität München, Munich \\ thomas.linner@bri.ar.tum.de \\ Georgi Temelkov, Maria Malinova, Dimitrina Jegova \\ Cand. Arch., Technische Universität München, Munich \\ dimitrina.jegova@mytum
}

\begin{abstract}
Bill Gates today announces the era of service robotics and estimates that service robotics as part of assisted environments will undergo a similar fast and rigid development as the spread of personal computers in private and economic areas since the nineties. Already in 1961 Joe Engelberger wondered if relegating robotic technologies to only industrial applications makes any sense: "The biggest market will be service robots." Today robots and distributed robotic subsystems actually start to permeate our every day surrounding home/town environment enhancing it with a multitude of services and additional performances. At the same time, this permeation is likely to dramatically transform our perception of robotic systems, robot technology, robots' performance and the environment they are becoming merged with. In order to make visible these transformations, the following article examines new application fields of robotics integrated with our living environment and related logical transformations on the basis of project works of an interdisciplinary course on "Ambient Innovation Robotics".
\end{abstract}

\section{KEYWORDS: Robotics, Physical Computing, Personal Assistance, Transformations}

\section{INTRODUCTION}

A close look at the fixed and loose components of our environments shows that all components of the housing environment are gradually integrated with electronics and micro-systems. "Miniaturization" and "Downscale" as basic forces of our technological development today enable a seamless integration of sensors, actors, control components and microelectronic systems into all subsystems, components and appliances. At the same time new approaches in computation link them to co-operating distributed systems and subcomponents which can respond actively or even proactive on certain use cases. Furthermore experts and masterminds as for example Bill Gates announce the era of service robotics and estimate that service robotics as part of assisted environments will undergo a similar fast and rigid development as the spread of personal computers in private and economic areas since the nineties. Already in 1961 Joe Engelberger wondered if relegating robotic technologies to only industrial applications makes any sense. "The biggest market will be service robots," (Engelberger, 1989) asserted Engelberger, who started the industrial robotics era when his firm (Unimation) delivered GM's first robot. Today, robots and distributed robotic sub-systems start to permeate our every day surrounding home/town environment enhancing it with services and additional performance. At the same time, this permeation is on the way to transform our perception of what robots are, robot technology, robots' possibilities and the environment they are merged with. This 
transformation which has to be understood as a natural part of the evolution of robotics will especially become visible when robots enter the field of service and assistance. Therefore, the following article examines new fields of application for robotics in assistive environments and accompanying transformations. All these examples are outcomes of an interdisciplinary course on "Ambient Innovation Robotics" which examined the complex interplay between permeating robotics, human beings, performance, technology, environment and form/appearance of future (robotic) artifacts.

\section{ROBOTIC SERVICE WALLS: IMMOBILE ROBOTS}

Today the independent and care supported living in a conventionally designed and low-tech home is often unpleasant, and in many cases not suiting to counterbalance or the ease of disabilities. Especially when entering a later stage of life, changes of habits and lifestyle occur and unforeseen disorders, multi-morbidity and disorder progresses, often make existing houses or flats inapplicable for elderly inhabitants. In many cases, a re-configuration of the existing home or the implementation of various assistive technologies as modular component systems would be needed to meet multiple needs with multiple sets of technologies. Normally, the implementation of new technologies, sensors, actors, assistance devices and robotic sub-systems needed in a certain use case normally is a complex, costly and time consuming matter often forcing elderly people to move to a new home, or to set aside the idea of being supported by advanced assistance technology.

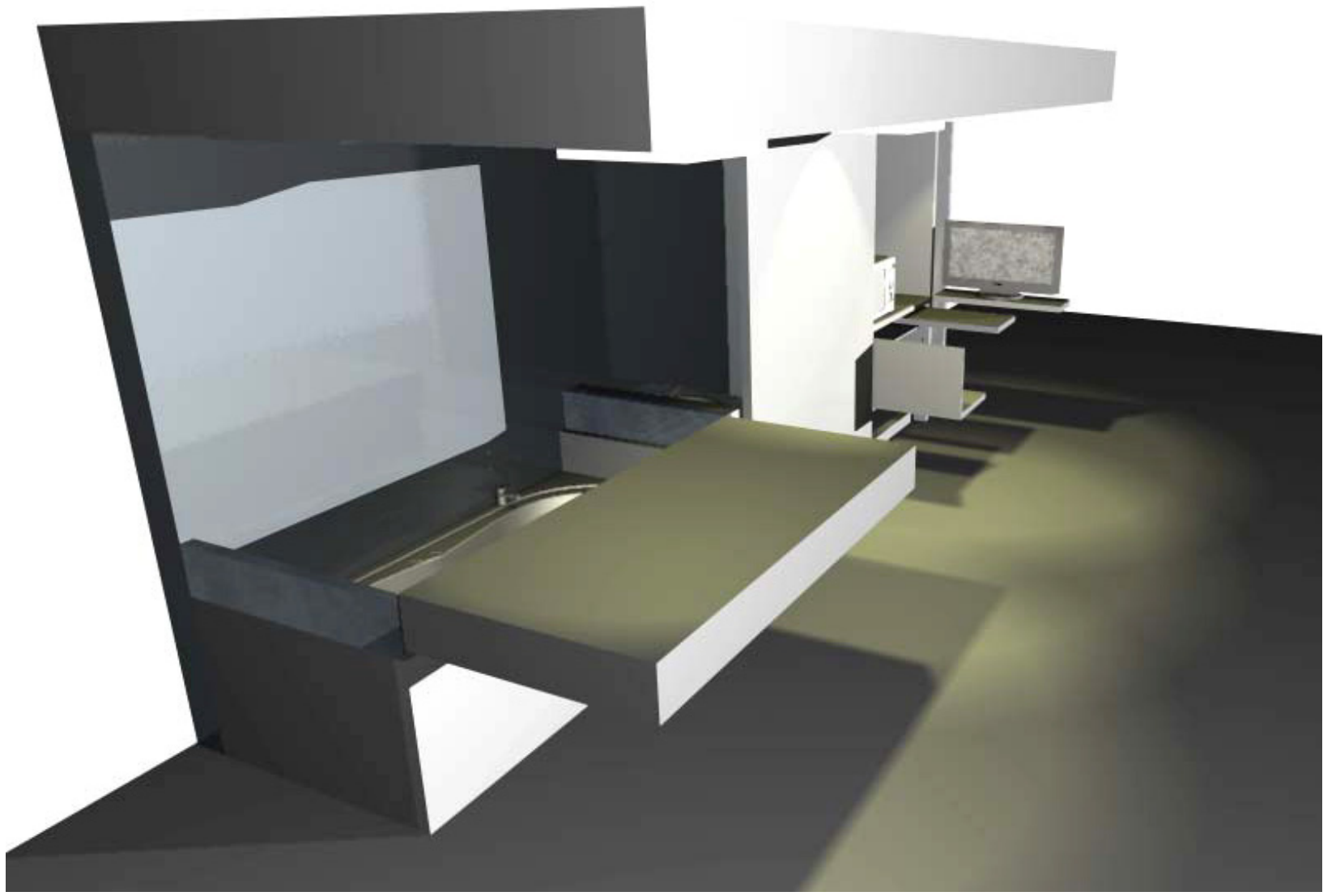

Figure 01: Highly compact robotic service wall 
The approach of robotic service walls/cores tries to simplify the integration of advanced assistance technologies into existing homes. The service core/wall is a compact, modular and customizable service entity, equipped with technology packages, bound together for supporting certain disabilities or sets of disabilities. The service wall/core contains all service functions and assistance technologies which thus do not have to be distributed over the whole flat. As only the service core/wall has to be installed in the home as a compact prefabricated and pre-configured module, cost and complexity of the rearrangement itself are minimized allowing more advanced and helpful assistance technology.
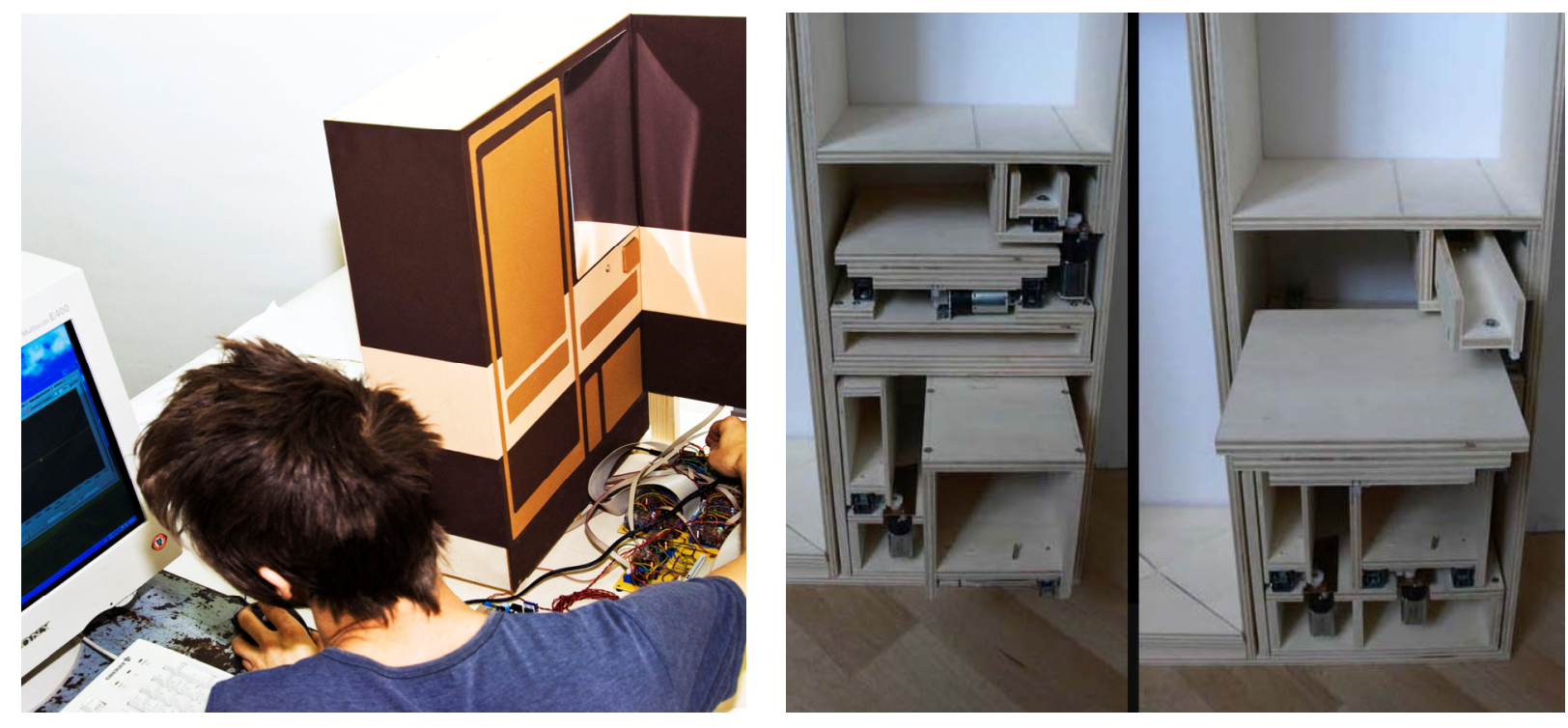

Figure 02, Figure 03: Fully functioning prototype model of service components, Scale 1:4

Robotic service walls/cores use of the concept of distributing and embedding various subsystems into environments. Environments enriched with "sensor" and "actuator" systems given certain autonomy, and able to control their complex internal functions can be considered as "ImmoBots": Immobile Robots (Williams et al., 1996; Roush, 2003).

\section{ROBOTIC FURNITURE}

Mechatronics systems, downscaled motors and lifting systems, integrated sensors and acorns in combination with new technologies for human-machine-interaction can be accounted as subsystems of immobile robotic systems. If such sub-systems are integrated and merged with everyday artefacts as furniture, appliances and whole buildings, those artefacts tend to change both, functionality and appearance. Emerging technologies are not only transforming functionality, but also designs and forms of our environment, following the principle of "form follows function" (Sullivan, 1896) being discovered and scientifically explained by L. Sullivan already more than one hundred years before. The integration of robotic systems and sub-systems is likely to transform the appearance of our environment and everyday's artefacts, similar as done through the implementation of new materials as steel or plastics once before. Figure 04 shows a study about an assistive kitchen, equipped with sensors and actors and with flexible and liveable multilevel workplanes. Those workplanes can automatically be adjusted to working tasks, people's height and ergonomic needs serving people and making cooking processes more efficient especially for elderly people. Moreover, new interface and projection technologies make it possible that information is not any more restricted to a separate screen but can be distributed over the whole system appearing on demand. Figure 04 shows that the whole kitchen 
system could be industrially fabricated as a modular kit. The study shows by using a simple example that, if the idea of integrating advanced assistance technology is worked out consequently, functionality and form of future artefacts as kitchens will distinctively be different in form than what we know as a today's kitchen.
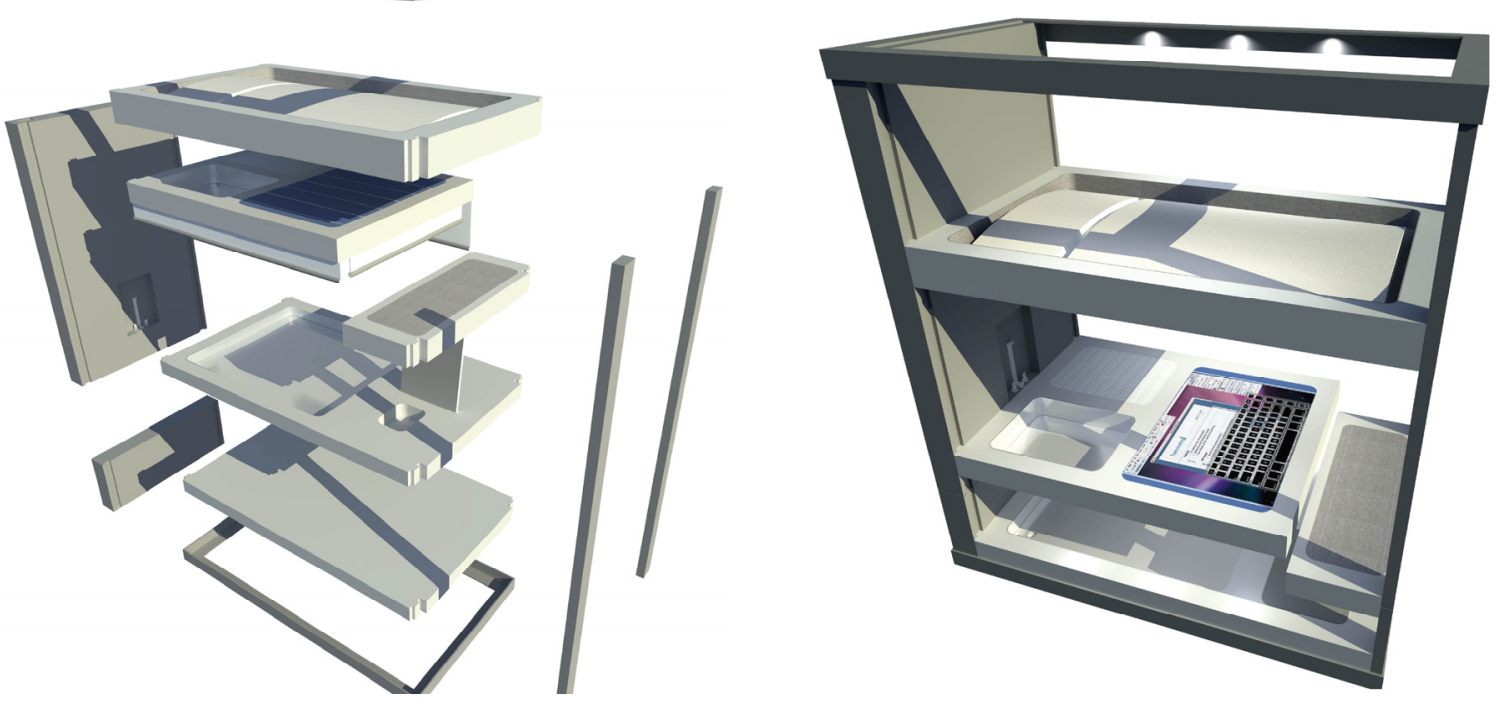

Figure 04: Modular and customizable robotic kitchen

\section{ROBOTIC CO-WORKERS AND ROBOTIC CARE ENVIRONMENTS}

In the future, technological complexity will be shifted from the care robot to the environment and distributed among various intelligent subsystems. The future care robot is supporting care helpers or people in need. It will be complementary part of an intelligent care environment as well as sensors and actuators attached to doors, furniture, ceiling and so on, to enable an environment to support and take over robotic tasks. The future care robot has the capability to cooperate with various subsystem components in its' surrounding melting robot and environment to an integrated and efficiently working entity. Figure 05 shows the concept for a multi-joint robot as subsystem of a robotic care environment. Some segments include adapters that allow the attachment of different tools and connectors in order to facilitate the nurse/ care helper aid with modular equipment. All elements of the care environment from bed to bathtub are designed as complementary part of the robotic care environment and have through plug and play interfaces the capability to couple with the robot informatically and/or physically to perform certain tasks as an entity. Figure 06 demonstrates the layout of a possible nursing station. A tablet storage (5), bathing area (1), four beds (3). The Nursing robots are performing different tasks, one is handing a patient a tablet (2), one is getting ready to move a patient (8) from the bed onto the netting (9) and one Nursing Robot (10) is charging its battery (6). The main idea in this scenario is to see robotic application and its surrounding environment as one organism built on cooperating and co-adapting subcomponents. In a robot supported nursing station various care robots can perform assistive tasks supported by intelligent subsystem components as for example bed, health care system, doors, toilet or shower being complementary elements of the whole system. 

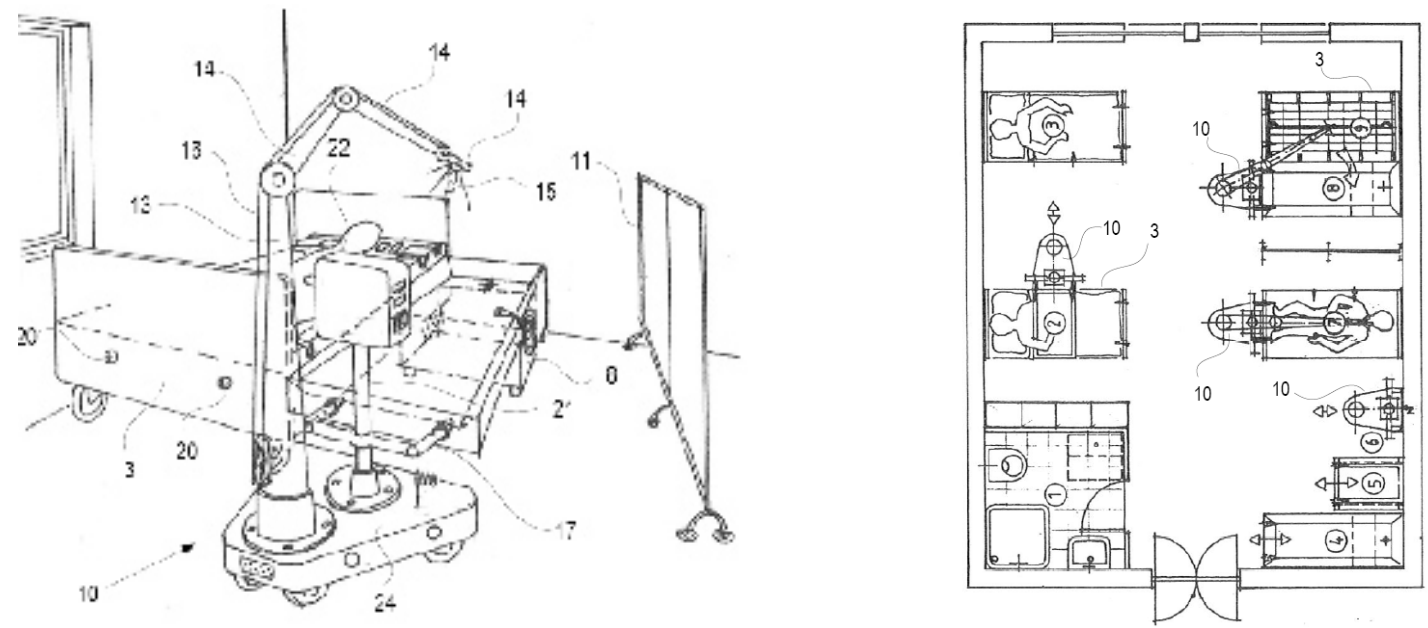

Figure 05: Robot as subsystem of a robotic care environment. Figure 06: layout of nursing station fully integrated with robotic subsystems

\section{MOBILITY ROBOTS}

E-Move is a system developed to help and to improve the quality of people's lives. It is strongly connected with today's issues and is designed to solve the problems of tomorrow. E-Move provides the ability to control one's own life, preserve self-respect, independence and maintains personal space, no matter the age group or health disability of the user. The system has three main components - a comfortable chair, a docking system and an electronic bracelet. Each is designed to match different people and to adapt to different situations in order to allow free movement both in private and social environments. The main goal of the system is the reintegration of people with health problems back into society. This reconnection will not only ensure higher quality of life-existence but will also develop these societies in all aspects, both economically and humanely.
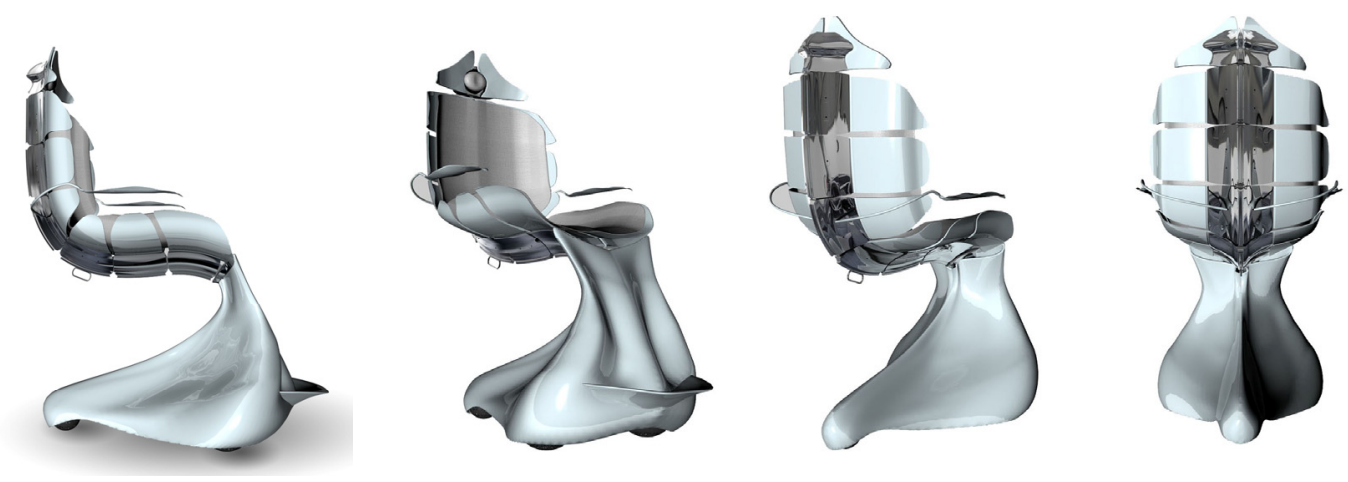

Figure 07: Concept for a mobility robot fusing to a high-performance entity with the user

The first component of the system is the seat. Its construction is inspired by the human spinal chord in order to be adjustable to all types of individuals. Every single vertebra is pre-fabricated and contains information, which is transferred between the user and the chair itself. An electronic system goes through the whole spinal cord. It serves the chair with ventilation, heating, and gives impulses when the user wants to stand up. It is like having a second, supporting spinal cord which follows the intentions and the movements of its user. The connection between the separate 
vertebrae is intended to be like the joints in the human body, which will allow the cord to move. The covering of the chair is also segmented in order to follow the spinal cord's movements accurately. The hand-, head- and feet-rest are all developed adjustable in order to be as much comfortable as possible. A docking system is designed to make the integration of the chair easier in almost all kinds of environments. The base is connected through the last vertebra-segment and can be easily removed when needed. The hardware that provides the movement for the chair is incorporated in an aerodynamically designed container. The innovative and ecological electricmotor can be easily charged at home. Such motors are state of the art, developed by Volvo and Toyota, which limit energy loss by converting breaking energy into electricity later to be used. The popularization and development of these types of motors will lead to the demand of battery station to become available in the city canters.
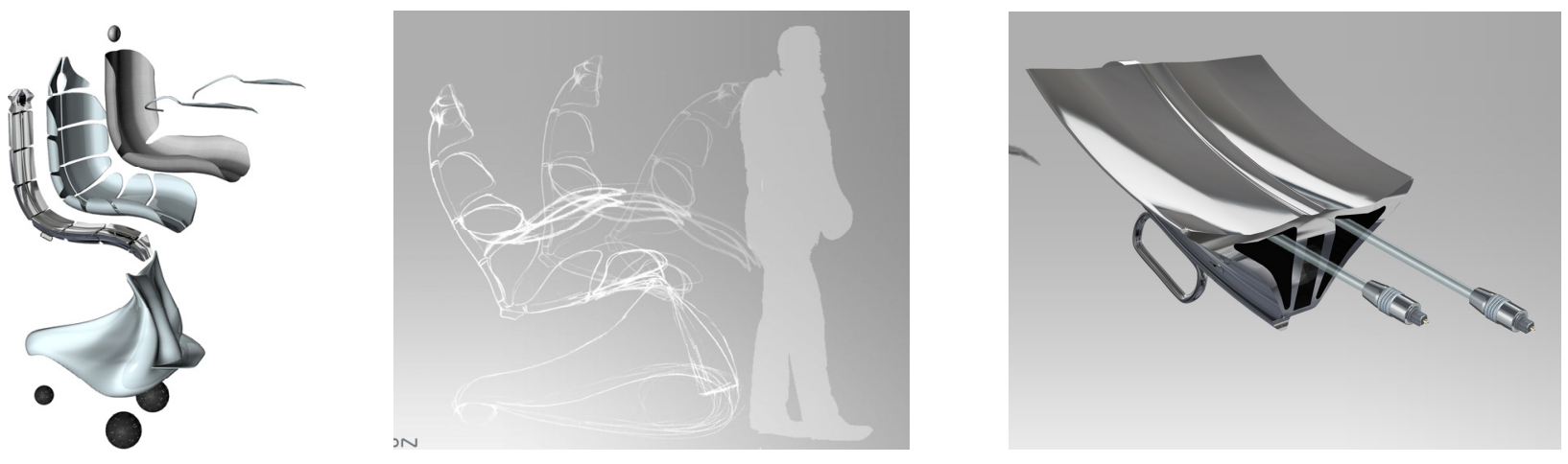

Figure 08: Modularity and ergonomics

The system can be used at home and on the street. If the user is also a driver the chair can be easily integrated into a car. The dock can be removed, and by a robotic arm the seat can be transferred into the car. In this case it also replaces the commonly used seat in the automobile and at the same time ensures individual comfort for the user. Climbing stairs is also made easier with this chair. As long as the staircase has special rails, the seat can just slid up and down. The third component of the system is the specially designed Bracelet. It is an electronic device, which performs the connection between the user and the surrounding world. It is multifunctional and controls and measures electronically all vital values such as pulse, blood pressure etc, which are displayed on the projection monitor. This information can be sent to the patient's family doctor, so the condition of the patient can be monitored at any time. Emergency medication can be stored in a compartment on the backside of the Bracelet. In an emergency situation the patient can react quickly and save his life.
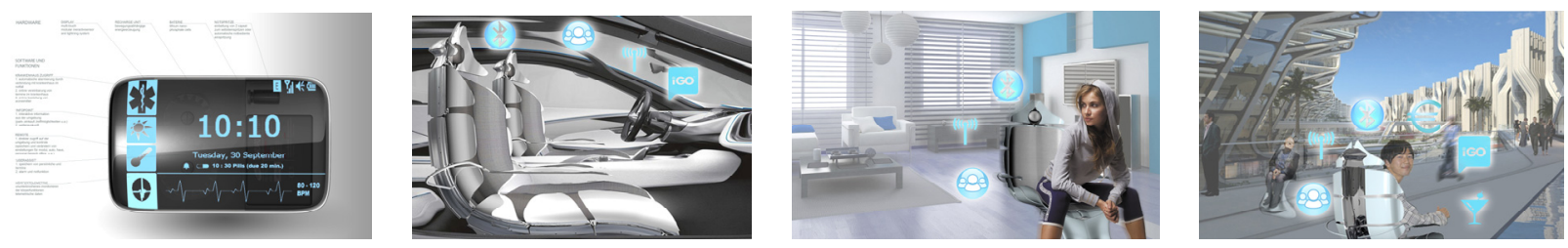

Figure 09: Examples for interaction with other users, car, house and city.

The Bracelet is also a communication device. Like a navigation system it receives all kinds of information about the city, the traffic, other users, even about the prices in the supermarkets. Thanks to the convenient user interface of the Bracelet all users can be connected through a global network, supported by satellites and local wireless signals making a faster and easier interaction with other users and the city. 


\section{ROBOTIC EVERYDAY COMPAGNION}

Accompanying "ubiquitous" mobility concepts as described in the former section a next step in the evolution of robotic assistants might be the introduction of robotic companions. Similar to Toyotas Robotic wheelchair which could be switched in a stand-by mode autonomously following its owner, robotic town companions could follow and assist with multiple activities in the town area as shopping, orientation, carrying of loads and/or guidance. Moreover, these robotic companions will not appear as typical technically looking or humanoid robots, but as simple and fancy artefacts which are used by younger people as well as by the elderly. The overall design principle enables its use for care receivers. Similar to our smart phones today, robotic town companions might assist us also with location based services, and a multitude of downloadable applications making robotics a fancy and widely accepted assistance technology.
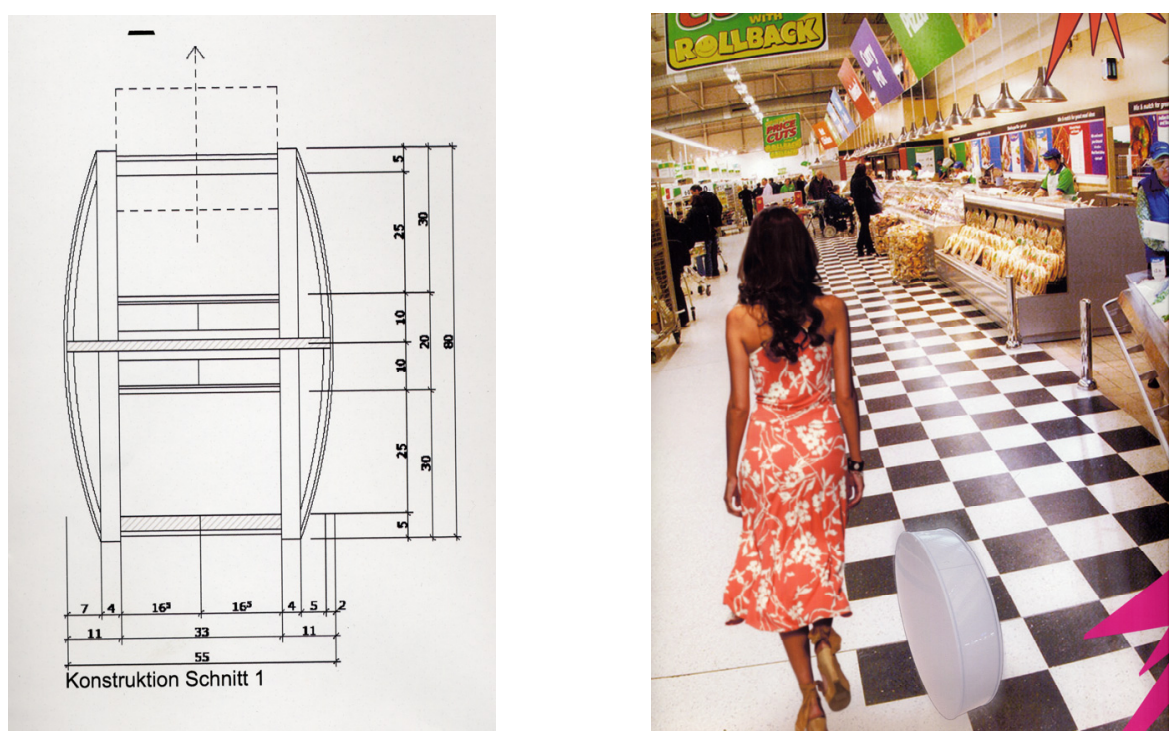

Figure 10, 11: Robotic shopping assistance

\section{CONCLUSION}

New application fields for robotics in the area of service and environmental assistance are generated through the tension between market pull on the one hand and technology push on the other. However, the integration of robotic systems and sub-systems is likely to transform the appearance of our environment and everyday artefacts similar as done through the implementation of new materials or technologies before. The integration and permeation of our environments and social spaces with robotic technology and related sub-systems will lead to new concepts and explore new forms as robotic walls, robotic furniture, robotic care environments, mobility robots and robotic everyday companions. Obviously emerging technologies are not only transforming functionality of upcoming hybrid robot-environment systems but also designs and forms of our environment following the principle of "form follows function" (Sullivan, 1896) being discovered and scientifically explained by L. Sullivan already more than one hundred years before. Despite all complex interdependencies and transformations, underlying principles as modularity, customization capability, system distribution, standardization and a well defined human-robot relation seem to be helpful tools in designing future hybrid robot-environment systems. Above that, modularity and standardization are important prerequisites for industrially fabricated and customized robotic enhanced assistive environments. Complex and multilevel high-tech environments will only reach affordability and long-term serviceability when fabricated with frontier industrial technology. 


\section{REFERENCES}

1. Bock, T. (2000) Robotic Gerontotechnology, Research Project for Japan Science Society, TU Munich, Germany, 2000

2. Bock, T. (1992) Life Support Sytem (LSS 3), Research Report for Japan Science Society, Prof. Thomas Bock, University of Karlsruhe, Germany, 1992

3. Bock, T., Linner, T. (2008) Service Oriented Design. Proceedings of $2^{\text {nd }}$ German Congress on Ambient Assisted Living, Berlin

4. Bock, T. (1988) Robot Oriented Design. Tokyo: Shokokusha

5. Bock, T., Linner, T. (2009) Adaptive Component Systems for Assistive Environments. Proceedings of $2^{\text {nd }}$ German Congress on Ambient Assisted Living, Berlin

6. Bock, T., Linner, T. (2009) Ambient Innovation Robotics. (AIR). Ed.: Technik in Bayern,VDI, Heft 4, Seite 37, Juli/August 2009.

7. Bock, T. (2010) TH Award Keynote: Turning Points in Construction. In: 26th ISARC 2009 International Symposium on Automation and Robotics in Construction, Austin/Texas, USA. Ed.: International Association for Automation and Robotics in Construction, I.A.A.R.C.

8. Calinon, S. Robot (1996) Programming by demonstration: a probabilistic approach. Lausanne: EPFL Press

9. Engelberger, J.F. (1989) Robotics in Service. Massachussets: MIT Press

10. Linner, T., Kranz, M. (2010) Robotic Service Cores for Ambient Assisted Living. Pervasive Health, Munich

11. Roush, W. (2003) Immobots take control. Technology Review, vol. Jan 2003, pp. 36-41,

12. Schraft, R.D., Volz, H. (2009) Service Robotics: Innovative Technology for service and supply structures. Berlin: Springer Science

13. Sullivan, L. (1896) the tall office building artistically re-considered. Essay

14. Williams, B.C., Nayak, P.P (1996): Immobile Robots: AI in the New Millenium. AI Magazine, NASA Research, vol 17(3), pp. 16-35. 1996

15. Picture 01: Cand. Arch. P. Graab, 2010

16. Picture 02, 03: Cand. Arch. F. Roedel, B. Ellmann

17. Picture 04: Cand. Arch. L. Mengel, S. Meyer, C. Müller, F. Müller, I. Muka, A. Myrenne

18. Picture 05, 06: G. Fabritz

19. Picture 07, 08, 09: G. Temelkov, M. Malinova, J. Dimitrina

20. Picture 10, 11: M. Bittmann, E. Lipod, M. Mayer, J. Schillinger, J. Schlenker, L. Schneider, S. Schürmann 